\title{
Radiation effects in insulators
}

\author{
A.M. Stoneham \\ AEA Industrial Technology, 424.4 Harwell, Didcot, Oxfordshire, OX11 ORA, UK
}

\begin{abstract}
My survey of radiation effects in insulators concentrates on the three main thrusts of mechanisms, materials, and applications. By mechanisms I include the processes occurring during irradiation, including defect production, amorphisation and mixing. This includes the roles of electronic excitation and of energy localisation, of charge redistribution, of surface processes like sputtering and desorption, and subsequent thermal processes. Materials must reflect new interests: the relation of the insulating oxides to oxide superconductors, and parallels between damage in organics and inorganics. Applications include cases where the radiation is the natural environment of space, the operating environment of a nuclear facility, or the controlled irradiation of an accelerator. In such applications the radiation effects are critical, and include insulator performance under radiation, and examples from erosion and lithography, adhesion, and coatings preparation.
\end{abstract}

\section{General introduction}

Insulators are a very varied class of materials. They include crystalline ionics, like halides and many oxides; the amorphous halide and oxide glasses; ice; the organics; nor is there a clear demarcation between insulators and semiconductors. This variety shows both in the radiochemical yield [1], the number of defects created per $100 \mathrm{eV}$ absorbed, and in the types of defect, e.g. reticulation versus scission in polymers, or photochemical versus ion displacement damage in ionics. There is a range of timescales in studies of radiation effects in insulators from the femtosecond phenomena to the geological. There is a range of radiations from photons to heavy ions. The radiation can be the natural enviromment of space, the operating environment of a nuclear facility, or the controlled irradiation of an accelerator [2]. In applications the radiation effects are diverse, from bulk processes to interface behaviour, and from thermal evolution to athermal reactions. The scientific basis in a meeting like this has both a basic common denominator of characteristic behaviour and a variety of contrasted behaviours.

\section{Mechanisms}

\subsection{What is special about insulators?}

There are significant differences between metals and non-metals, and these affect the radiation response [3]. The first comes from the fact that most non-metals contain more than one species of atom, so that $\mathrm{NaCl}$ contains $\mathrm{Na}$ and $\mathrm{Cl}$, whereas $\mathrm{Cu}$ metal involves just $\mathrm{Cu}$ atoms (though clearly there are exceptions, e.g. diamond on the one hand and ordered alloys on the other). There are then effects simply because of the different masses of the components, e.g. $\mathrm{Cl}$ and $\mathrm{Na}$ have different masses. More importantly, there is a strong rule which asserts that defects in ionics tend to keep each species on its own sublattice. Thus $\mathrm{Na}$ tends to remain on the $\mathrm{Na}$ sublattice, since the Madelung energy is large and opposes cations on anion sites; when there are several cations, ionic radius issues tend (much more weakly) to keep them on their own sublattice. Clearly there are exceptions, examples being:

(i) In the radiation damage of alkali halides, perfect dislocation loops form by a process in which neutral halogen molecules occupy cation/anion divacancies (a remarkable defect which is essentially unobservable); this can be regarded as an antisite defect with a halogen at a cation site [4].

(ii) In III-V semiconductors, antisite defects can be formed with relatively low energy. This affects strongly dislocation climb, since simple defect reactions can transfer disorder from one sublattice to the other (e.g. $\mathrm{Ga}$ interstitial displaces As to form an As interstitial and a $\mathrm{Ga}$ antisite) without the need for the complex mechanisms once assumed [5]. We shall sce later that antisite defects near the surface can affect stimulated desorption too, through the effect they have on energy localisation.

(iii) In $\mathrm{UO}_{2}$, one of the key fission products is iodine. In addition to the obvious sites, $\mathrm{I}^{+}$is stable at the cation site in certain situations [6], not just the obvious $\mathrm{I}^{-}$at the anion site. 
A second difference between metals and non-mctals is that defects in insulators can have a range of possible charge states $[3,7,8]$. For ions in oxides like $\mathrm{MgO}$ or strontium titanate, transition metal dopants can exist in three or four charge states, and equilibrium takes some time - even days - to establish. This has several consequences, one being that electric fields are set up which can themselves modify defect evolution (see also section 5.1). But there are other effects too, especially near to interfaces between media with different dielectric constants, where the existence of charged defects is one of the routes to radiation-enhanced adhesion (section 5.2).

A third difference between metals and non-metals is that excited states can last long enough to matter. Moreover, the energy needed to produce an excited state is finite, unlike a metal. The minimum energy to create an electron and hole is the band gap, though for most radiation about three times this energy is needed per electron-hole pair. For semiconductor detectors, there are very special circumstances (e.g. when addressing controversies like whether or not the neutrino has mass) where it is important that some of the energy is taken up by plasmon excitation, without generating electrons and holes subsequently [9].

Optical excitation can be sufficient to generate vacancies and interstitials. When the band gap is large enough, there are several routes to localise much of this energy and to use it efficiently in displacing atoms. The damage mechanism in alkali halides, for instance, involves the self-trapped exciton. Just how the process occurs is still controversial in part, but the outline is clear [10-12]; an exciton is formed, self-traps (and so localises distortions and energy) and then evolves into a separate neutral anion vacancy ( $F$ centre) and interstitial (H centre) separated along a close-packed row.

Subsequent reactions can also be affected by electronic excitation, either because different charge states have different diffusion rates, or because electronic excitation itself affects jump probabilities. Excited states can last for long enough for spectroscopy, and this is a powerful source of information on the damage mechanism.

Optical properties are, of course, a powerful source of information about the defect populations which emerge under irradiation. Thus in sapphire, it appears there are no dense collision cascades, contrary to metals like $\mathrm{Al}[13,14]$. This may be because the energy for maximum displacement cross-scction is different or because of the higher thermal activation energies for the oxides. More defects survive, so that optical measurements of defects (primarily the Al vacancy in sapphire) can be used as a damage monitor, operating up to $310^{\circ} \mathrm{C}$. Interestingly, damage efficiencies are similar for ion-beam and neutron irradiation; after allowing for the temperature dependence of the damage effi- ciency, the swelling appcars to depend predominantly on the displacements per atom.

\subsection{Thermal processes}

What roles are there for the thermal and other slower processes after the primary event? One role is clearly defect reactions: the way that dislocation structures develop, the way that anion vacancies evolve into metal colloids, the way that carbonaceous regions emerge in irradiated polymers or graphitic regions in diamond-like carbons.

Clearly thermal processing will usually tend towards some sort of equilibrium. But this equilibrium may be inaccessible without radiation processing, e.g. when ion implantation introduces concentrations far in excess of thermodynamic solubility limits. An interesting example here is the production of gold nanocluster composites by implantation of silica. The fact that high gold loadings can be achieved means that the effective nonlinear optical susceptibility is 200 times larger than for ruby-gold melt glass [15].

Since radiation methods can operate at low temperatures, radiation processing can avoid those higher temperatures at which critical component phases degrade. An example is diamond-like carbon coatings on polymers, formed by radiation effects on a hydrocarbon layer [16]. I have often hoped that radiation effects would yield special oxide superconductors, as phases which cannot be obtained by standard ceramic technolngy hecause the special phase is not stable at the normal processing temperatures [17].

A second role for radiation is what one might describe as "stirring". The complex oxide glasses (e.g. silicate glasses with other elements added to give them desired properties, or simply to immobilise the added elements if they are radioactive) have "ideal" densities which depend in a very systematic way on the composition. There is clearly a fairly well-defined quasi-stable state, even though the glass is arguably not in equilibrium. In fact the density is one which corresponds roughly to oxygen close-packing [18]; this, in turn, implies medium-range order, as has recently been identified in other ways. But there is an interesting application to radioactive waste glasses, where there may be nearly 100 elements incorporated. How will the density change with time once the glass leaves the chemical plant in which it is prepared? Will this alter as the waste changes in composition? The rule which emerges [19] is this: the waste glass will move with time to the "ideal" density, as if the only effect of radiation were to speed equilibration (hence the term "stirring"). Moreover, observation confirms too that the rate at which the "ideal" density is reached depends systematically on the glass composition, correlating with the viscosity dependence. 
A third feature concerns stored energy. In an ionic solid, the high Coulomb energies allow there to be rather high amounts of stored energy. In silica, for example, Antonini et al. [20] have found an order of 1 $\mathrm{eV}$ per molecular unit. Is this credible? It is actually well in line with what one would expect from a compilation [21] of enthalpies of various crystalline and noncrystalline silicas as a function of molecular volume. Both the crystalline and amorphous versions have enthalpies which are broadly similar, but displaced; if they are regardcd as parabolic functions of molecular volume, the curvature is of the order of that expected from the compressibility of vitreous silica. But the amorphous forms, apart from having lowest energy for a volume some $25 \%$ larger than quartz, have an energy at constant volume some $0.25 \mathrm{eV}$ higher too. This suggests a stored energy of a few tenths of an $\mathrm{eV}$ per molecular unit is both credible and in line with thermodynamic data.

Whether this is true in other systems too needs clarification. In the case of self-damaged $\mathrm{CaPuTi}_{2} \mathrm{O}_{7}$, one term is the elastic strain energy from the volume misfit between disordered inclusions and the crystalline matrix. The decrease in strain energy accompanying redamage of the metamict ceramic [22] shows that this elastic energy contributes only $20 \%$ of the observed energy decrease. Clearly, other types of disorder are involved.

\section{Radiation types}

\subsection{General issues}

We may divide the phenomena into two broad groups: those which transfer energy to the nuclei, and those which transfer energy to the electronic system. Several basic questions arise. Firstly, when can we use a "billiard-ball" model (or, more strictly, some interatomic potential) and ignore explicit consideration of electrons [23]? Secondly, when can we assume that primary events are random, and ignore effects of preexisting disorder on displacement energies, etc.? Thirdly, at least when the electronic system is excited, there is the question of energy localisation, since the simplest band picture always implies delocalised electrons. Exchange of energy between electrons and nuclei is inhibited by the large mass discrepancy, but is an important compoment of radiation damage. The transfer is primarily one way, with electrons driving nuclear motion. Transfer of nuclear energy to electrons does occur (it is of some importance in plasmas) but is less clear in its consequences [24].

This division is simplistic in scveral ways, not least because subsequent processes can be important: excitation by secondary particles, stabilisation in metastable states, inhibition of recombination by electric fields, etc. If we are dealing with one species only, much of the behaviour is described by Lindhard's rules [23]. To the extent that potentials can be characterised by Lindhard's parameter $m$, there is a high degree of universality of behaviour. But even when a billiard-ball picture can be used, the sequence of events can be complicated. One complication concerns the size of the region over which energy is deposited. Clearly there are general features: electrons produce sparse damage, with widely separated point defects dominating; heavy ions produce clusters of damage and the debris of cascades. Likewise, the length scale for deposited energy is related to the path length, which itself scales in energy as a power $2 m$ of energy $E$; from this one can deduce how the maximum temperature (defined by the host atom kinetic energies) will vary with $E$ and $m$. There are separate timescales for the projectile slowing, for the recoil atoms to set up an ion temperature, and for these hot ions to thermalise.

What implicit electronic effects might one expect? For example, is there really a threshold corresponding to the electronic band gap? Is the average electron density really what determines stopping, or are there effects related to the formation of bonds? There seems no evidence for such a threshold, and the effects of bond formation, whilst discernable, are modest [24].

\subsection{Energy localisation}

How does energy localisation occur? We should consider three cases [12]. Firstly, there is core excitation. Here the excitation can remain localised for a period long enough for some defect process to occur; correlation in a band with very small bandwidth is a factor [25]. Secondly, there can be self-trapping, in which the strong electron-phonon coupling drives a local distortion. This is especially important [26]. One key question in some systems is whether or not the localisation occurs at a special site (perhaps some precursor already exists) or not. The several proposed excitonic methods of defect generation are in this class. Thirdly, the localisation may involve an impurity.

From the many defect-associated cases, we may look at three examples. The first is $\mathrm{CsI}: \mathrm{Na}$, an $\mathrm{X}$-ray phosphor which exploits the large cross-sections of the two species Cs,I with high atomic number. The substitutional $\mathrm{Na}$ is amphoteric: it will trap electrons, an effect which is mainly electronic, associated with the electronegativity of the $\mathrm{Na}$. It will trap holes too, but the effect is mainly elastic associated with the different ionic radius and the self-trapping distortion of the free holc [27]. The emission is apparently from an exciton trapped by the impurity; kinetics show that either electron transport or exciton transport is involved. 
The sccond example concerns transition metal impurities. A good example is $\mathrm{Ce}$ in glasses, where it is included to minimise darkening. If a glass is exposed to gammas, the Compton "wind" tends to transfer electrons in the direction of the gamma flux. Ce ions near the irradiated surface tend to become $4+$, and those further "downwind" become $3+. \mathrm{Ce}$ is chosen because the optical absorption of both charge states is at an energy sufficiently high to minimise loss of transparency. Perhaps the most important aspect is the development of a significant electric field from this charge transfer, one which has implications for this and other systems. In a multilayer system such as AlAs/ GaAs [28], where the doping of each layer can be controlled, the electric fields can have a profound effect on damage cascades produced by heavy ions ( 320 $\mathrm{keV} \mathrm{Ga}^{+}$in this case), raising the possibility of steering unwanted damage to regions of a sample where they are less important. The drift of implantation damage in this case is driven by the field associated with discontinuities in the band structure and in defect enthalpies. Large electric fields alone can generate defects too. This has become increasingly relevant with scanning tunnelling microscopy. However, the fields are far larger than in the degradation effect, and they are also spatially inhomogeneous. Such fields are important in so-called nanoswitching and some nanowriting phenomena.

The third example relates to the role of muons, which can be regarded as both radiation and a probe of consequences [29-31]. The unexpected result is that there appears to be luminescence associated in some way with the muon. The luminescence has some resemblences to that from the self-trapped exciton, though there are some problems. In particular, the luminescence occurs after the muon has decayed. One possibility notes that the energy surface of the self-trapped exciton is flat, probably with several minima. Could it be that the muon "catalyses" the exciton into one of the metastable minima?

\subsection{Other aspects of electronic mechanisms}

The removal of atoms or ions from surfaces can proceed through electronic processes. Energy deposition is followed by localisation, whether of energy or momentum or both; ionic motions then lead to emission, followed by processes in the vapour phase [32]. Vapour phase processes may involve electron transfer (perhaps neutralisation of an ion) or breakup of some aggregate. The emission processes are of two main types. The Menzel-Gomer-Redhead mechanism involves excitation into an antibonding state; this is analogous to the "local excitation" mechanism of recombination-enhanced diffusion, or to the excitonic mechanisms of defect generation in halides. The laser-in- duccd desorption of Al from sapphire appears to proceed by this mechanism [33].

The Knotek-Fiebelman process [34], more like the Bourgoin-Corbett mechanism of enhanced diffusion, prepares an ion like oxygen, normally an anion, in the opposite charge state $\mathrm{O}^{+}$. When a core electron of a maximal valence ion (say $3 p$ of $\mathrm{Ti}^{4+}$ ) is excited, de-excitation involves the loss of two or more electrons from the nearby anion. The switch of charge leads to Coulomb forces which drive emission. This process has been proposed recently for desorption from very thin layers of alkali halides $(\mathrm{NaCl}, \mathrm{KCl}, \mathrm{KBr}, \mathrm{KI}$ and $\mathrm{CsI})$ on $\mathrm{W}(110)$. Post-emission neutralisation is important [35].

One recent example of interest shows that electron-hole pairs on the 110 surface of GaAs are especially effective in the defect-initiated emission of neutral $\mathrm{Ga}$ atoms. This shows in part through the enhanced yield when the photon energy increases across $1.87 \mathrm{eV}$ and across $2.55 \mathrm{eV}$ [36]. Nor should one ignore the influence of structural defects. Laser ablation of $\mathrm{GaP}$ (110) appears to be initiated by vacancies, and there are two discernable components, one whose yield falls rapidly with the number of laser shots (type A, ascribed to adatom initiation), the other whose yield falls more slowly (type $S$, ascribed to kink-type defect initiation) [37].

Sputtering of very thin films can give useful insights. Most sputtering models are based on the assumption that there are two types of process: those which determine atomic motions in the bulk, and those processes at the outermost surface which give emission. $\mathrm{MeV}$ ion sputtering of thermal oxide on silicon shows a dramatic decrease in sputter yield for films less than $2 \mathrm{~nm}$ thick [38]. The results are consistent with the process being mainly a near-surface phenomenon, not entirely at the surface. Whether there is a component associated with electronic excitation processes is not certain. Like many other insulators, $\mathrm{SiO}_{2}$ desorbs ions under electron bombardment, with a threshold corresponding to core excitation [39]. Silicon dioxide does readily show desorption under optical excitation; very thin oxides become unstable at raised temperatures after laser irradiation, losing oxygen [40].

Fast ions can eject large organic molecules (e.g. the bovine insulin molecule) intact, even those which would decompose on heating [41]. The ejected species are weakly bound, so that relaxation available in various processes suffices to give sufficient kinctic cncrgy. Onc component of the process relates to track formation, in that there is a central positively charged core from which electrons have moved, and the Coulomb energy gives rise to a shock wave. A heavy incident ion of say 1 $\mathrm{MeV}$ produces a highly ionized central zone of perhaps $0.4 \mathrm{~nm}$ radius. Large intact ions come from a zone between 1 and $2 \mathrm{~nm}$ from the centre of the impact; 
large neutrals arc ejectcd from the further region to about $4 \mathrm{~nm}$; beyond that, there is damage to about 10 nm.

Laser ablation is less-well understood. One recent analysis argues that an important factor is the way that a surface vacancy makes it casicr to remove atoms adjacent to that vacancy, so that there is a high power dependence of the yield on laser fluence. If true, this illustrates a case of non-random defect generation [42].

\section{Materials}

\subsection{Insulating oxides}

Excitonic mechanisms of defect production are well established in halides. Evidence for similar processes in oxides is strong, but it is less clear what happens. Certainly there can be carrier localisation, and often energy localisation as self-trapped excitons [26]. One case where more is known is the self-trapped exciton in quartz $[43,44]$, where recent calculations show the hole component localises on an oxygen and the elctron on a silicon, with a substantial motion of the oxygen from its original site. The distortion is such that the exciton emission is around $2.6 \mathrm{eV}$, far less than the band gap of nearly $10 \mathrm{eV}$. The characteristic blue luminescence is seen in all silicas, including those silica glasses used in optic fibres. Recent optical experiments confirm many of the features of self-trapping and the similarities of the relaxed structure to a transient Frenkel pair [44]. A survey of defects observed in such glasses confirms that the defect species are usually closely similar to those in crystalline silicas $[45,46]$.

Two defect types which are common in oxide systems are the oxygen vacancy in one of its charge states, and the peroxy radical, with two oxygens bound to each other [45]. Thus the oxygen vacancy in quartz observed is a $\mathrm{Si}$ dangling bond ( $\mathrm{E}_{1}^{\prime}$ centre, the positive charge state), and has the $E^{\prime}$ centre as its analogue in amorphous silicas. The peroxy can be thought of as a form of oxygen interstitial. In close-packed oxides like $\mathrm{MgO}$ the peroxy is seen only when other defects leave space for it; in oxides like silica and telurium dioxide, the peroxy is more readily formed.

The formation mechanism for $\mathrm{E}^{\prime}$ centres shows evidence for pre-existing defects in vitreous silica [47]. There are two processes: the X-ray activation of preexisting structural defects, and the $\mathrm{X}$-ray creation of similar defects. There are far more $\mathrm{E}^{\prime}$ precursor defects in "dry" Suprasil-W1 (which has less than 2 ppm $[\mathrm{OH}]$ than in the "wet" Suprasil-1 (with about 1200 ppm [OH]), yet the new defects are more easily formed in the wet material. There is a strong corrclation of radiation hardness (rate of producing damage) with $\mathrm{OH}$ (and also on dose and thermal history) but no identified correlation with spin signals. Other work suggests that $193 \mathrm{~nm}$ excimer irradiation encourages the reaction between two silanol groups, leaving a stretched bond which is susceptible to $E^{\prime}$ formation [48].

When buried oxide is formed in silicon by oxygen implantation to large doses (SIMOX) $\mathrm{E}_{1}^{\prime}$ centres form only in oxide precipitates. However, there are $\mathrm{E}_{1}^{t}$ precursors in the buried oxide layer, and these can be activated by electron or gamma irradiation [49]. In MOS devices too there is also latent, thermallyactivated interface-trap formation, again involving hydrogen motion [50,51]. A two-step process is proposed: (i) radiation-induced holes release $\mathrm{H}$ as they move through the bulk of the oxide; (ii) hydrogen ions move to the $\mathrm{Si} / \mathrm{SiO}_{2}$ interface under an applied positive bias. The effects of radiation on MOS (metal oxide semiconductor) devices are enhanced by the large difference in mobility between electrons (perhaps 10 $\mathrm{cm}^{-2} / \mathrm{V} \mathrm{s}$ ) and holes (less than $10^{-6} \mathrm{~cm}^{-2} / \mathrm{V} \mathrm{s}$ ) in $\mathrm{SiO}_{2}$. The electrons can move rapidly to the electrode, leaving an excess of relatively immobile positive charge. The holes give both a space charge and take part in local defect processes, either at interfaces or, it is argued, providing precursor sites for defect generation [52].

One important theme in the evolution of defect species concerns what the mobile species actually are. A particularly useful analysis of defects in silicas [53,54] shows the consequences of different mobile species in different ranges of anneal temperatures. Whilst the precise temperatures depend on water content, etc., the broad features are these: At the lowest temperatures (say below $120 \mathrm{~K}$ ) after X-irradiation neutral hydrogen atoms alone are mobile; hydrogen molecules move readily in the higher range up to room temperature. In gamma-irradiated silicas, molecular oxygen diffusion $(400-540 \mathrm{~K})$ and water molecule motion (540-750 K) are the important species. Only at higher temperatures still do intrinsic defects move. The various mobile species interact with existing intrinsic defects, like non-bridging oxygens or $E^{\prime}$ centres.

The radiation enhancement of noble gas diffusion in $\mathrm{U}_{3} \mathrm{O}_{8}$ shows both the value of modelling and some general points in the behaviour of complex oxides under irradiation [55,56]. There are two structurallydistinct oxygens in $\mathrm{U}_{3} \mathrm{O}_{8}$. Those in the $\mathrm{U}-\mathrm{O}$ planes have easy vacancy formation and low migration energy. Those in the -U-O-U-O-U- chains normal to the planes are harder to form thermally, with an energy cost perhaps $2 \mathrm{eV}$ larger than for the plane oxygens. Vacancies in the chains are readily formed under irradiation, but their population is fairly stable, so amorphisation occurs at low doses. It would be interesting to assess whether amorphisation is more widely associated with two or more populations of one ionic species, 
with defccts of one population more readily formed. But this also resolves the apparently inconsistent results for Xe diffusion: when thermal vacancies dominate, a large activation energy is expected $(3.75 \mathrm{eV})$ and seen $(3.9 \mathrm{eV}$ [57]); when there is a radiation-induced population of chain vacancies, smaller energies are predicted (1.3 eV) and observed (0.85 eV [58]).

\subsection{Oxide superconductors}

The oxide superconductors are so striking in one aspect of their behaviour (the superconductivity itself) that one might expect them to be very different from conventional ceramic oxides in other respects. Yet, as a recent review showed [17], the superconductors are strikingly similar to other oxides in many respects. One example is radiation damage. To be sure, the damage can affect the superconductivity, either beneficially by pinning flux lines, or detrimentally in many other ways. But other features are as one would expect. It is the oxygen which is mobile, and from which the major effects arise as it is redistributed. Perhaps partly because most of the action occurs on just this oxygen sublattice, there is approximately "universal" behaviour: the change in critical temperature per unit of fluence is linear in nuclear energy deposition [59]. However, more may be involved; certainly, amorphisation appears to start with the rare earth sublattice, and there is certainly cation reorganisation in the formation of new perovskite phases.

\subsection{Organics}

There are some interesting analogies between irradiation effects of oxide superconductors and insulating organics. The important feature in both cases can be the dissolved interstitial oxygen, which can then react with damage $[3,60,61]$. The standard effects of radiation (including ultraviolet) on polymers are scission (in which random breaks in the chain occur, rapidly reducing chain length) and depropogation (which generates the monomer, as in polymethylmethacrylate). The effects of increasing dose show a range of phenomena $[62,63]$ For $2 \mathrm{MeV} \mathrm{Ar}^{+}$ions, for example, doses of $10^{+10} / \mathrm{cm}^{2}$ and above show basic beam-induced structural effects; polymerization of monomers or dissociation of polymers. Doses above about $10^{+11} / \mathrm{cm}^{2}$ begin to indicate scission or cross-linking, and basic lithographic processes (in lithography, it is the smaller units which are dissolved away, so cross-linking gives negative resists, where the irradiated region is less soluble; scission gives a positive resist since the irradiated regions are more easily removed). Oxygen becomes especially important when doses exceed $10^{+12} / \mathrm{cm}^{2}$. For still higher doses, above $10^{+13} / \mathrm{cm}^{2}$, carbonization occurs, with associated effects on electronic transport [64].
It is the carburization which appears to be associated with the insulator-metal transition in both polymers and diamond-like carbon. In essence, the radiation leads to regions of carbon from which hydrogen has been released (whatever its fate); the carbonaceous rcgions are relatively conducting, likc graphitc. As the dose rises, the conducting regions overlap until there are percolation paths through the polymer, so it is macroscopically conducting. There is here scope for descriptions in terms of Poisson distributions of local damage, normally expressed in terms of tracks with a defined radius $[65,66]$.

As well as breakup of polymer chains, there can be cross-linking of chains, which can yield a more brittle structure (see also later in relation to enhanced adhesion). Roughly speaking, cross-linking is favoured when the chain carbons are linked to $\mathrm{H}$, whereas degradation occurs when they are linked to other $\mathrm{C}$ atoms. Thus cross-linking dominates in polyethylene, polystyrene, polymethylacrylate, and polyvinyl chloride; it increases the cohesion and resistance to chemical attack of the polymer, and may also lead to shrinkage. Degradation dominates in polyisobutylene, polyalpha-methylstyrene, polymethyl methacrylate and polyvinyledene chloride; gas (e.g. hydrogen) may be evolved too. However, it is the presence of oxygen which is especially important: the free radical $\mathbf{R}-$ becomes ROO-; this reacts with $\mathrm{R}^{\prime} \mathrm{H}$ to give $\mathrm{ROOH}$ and $R^{\prime}$ - and degradation can continue. In many cases, degradation is limited by molecular oxygen diffusion [60]. The value of antioxidants is widely exploited.

As an example, argon plasma treatment of teflon surfaces in the presence of oxygen leads to peroxy radicals bonded to carbon in the cross-linked structure. Thus there are components like $-\mathrm{CF}_{2}-\mathrm{CF}(\mathrm{OO})-.\mathrm{CF}_{2}-$ [67]. The cross-linked peroxy radical can be converted into the chain-scission peroxy radical by UV irradiation and exposure to the atmosphere. The increase in oxygen content and decrease in fluorine content make the surface more easily wetted by water. The oxygen which modifies the polymer can also be atomic. Thus for space applications, including polyimide pitting in cargo bay of low altitude orbiting space shuttle, it is atomic oxygen with energy of about $5 \mathrm{eV}$ which is important [68].

Microwave water plasmas (as proposed for various medical and microelectronic applications) also modify polymer surfaces. Thus in a combination of processes (adsorption, desorption, random bond breakage perhaps by $O\left({ }^{3} \mathrm{P}\right)$, surface diffusion, hydroxylation, removal of volatiles, etc.) the polyimide forms hydroxyl species, and the surface morphology changes. Both changes contribute to changes in adhesion behaviour [67].

Photoablation by excimer laser irradiation $(248 \mathrm{~nm})$ appears to lead to another type of change. Conical 
defects appear (rather than the pits from etching) and the ablation rate diminishes with time. What seems to be happening is that the polymer "radiation hardens": regions which are more carbonaceous are less easily ablated, and initiate cone formation [69], as one would cxpect from analogies with refs. $[65,66]$.

\section{Applications}

\subsection{Insulators for fusion: radiation-induced conductivity}

One puzzle to emerge in the study of insulators, like alumina, for fusion reactor applications, is a form of radiation-induced electrical degradation [70-76]. Virtually every aspect of the heating, control and plasma diagnostics relies on the insulator. Degradation in alumina occurs much more rapidly when there is displacement damage, ionizing radiation, and an electric field (RIED: Radiation induced electrical degradation). The puzzle lies especially in the role of the electric field: even small fields $(<1 \mathrm{kV} / \mathrm{cm})$ somehow affect damage mechanisms so that large disc-like defects are formed and the resistivity degrades. There is evidence that more vacancy defects are formed, and that these enhance $\mathrm{F}^{+}$above the neutral anion vacancies $\mathrm{F}^{0}$. Whilst the explanation is not completely clear, some possibilities can be noted. First, ionizing radiation generates electron-hole pairs, and these are separated by an electric field so that all do not recombine. Secondly, there are mechanisms which allow fields to build up in low-symmetry crystals, and the actual internal fields can be quite large. Ionization also yields transient defects as well as electron-hole pairs. The electrons and holes are themselves affected by electric fields, which shows in the luminescence attributed to selftrapped excitons [77-79]. The explanation in this case is the Onsager effect, where the field discourages recombination of an electron with its hole in favour of separating the carriers and recombination at luminescent centres. Presumably the internal field inhibits the recombination of charged vacancy/interstitial pairs, though there may be other effects too. In RIED, the process may be more to do with the differences in diffusion rates on the two sublattices and the extent of defect aggregation (for example the bias of a defect aggregate towards capturing other vacancies versus capturing interstitials). Certainly RIED and the formation of anion vacancy aggregates occur most readily at essentially the same temperature, $450^{\circ} \mathrm{C}$; the cation defects diffuse less rapidly at this temperature. Just how the combined electric field and irradiation influence this is not obvious. Nor is the nature of the so-called "aluminium" precipitates clear, since diffraction data show these are not simply fcc aluminium [80]. What is needed is a fuller treatment of the evolution of the microstructure, extending the earlier analyses $[81,82]$ to include the role of the field. The consequences of an evolving microstructure are evident in a range of damage phenomena; another example of some interest is the way the conductivity and its temperature dependence evolve with the implantation of iron into $\mathrm{MgO}$ [83].

Surprisingly, there is no RIED effect in vitreous silica. This raises the question of whether the structure of alumina plays a part; for example, if the small applied field could be "concentrated" by some low symmetry defect (perhaps a stacking fault or related) its effect could be more obvious. Ruby ( $\mathrm{Cr}$-doped alumina) does indeed show some unusual behaviour after laser pumping in an electric field [84], with domains of strong internal electric field $(350 \mathrm{kV} / \mathrm{cm})$ along the $c$-axis, different regions having different signs of field. The behaviour is related to the axial polar symmetry of defects in alumina; phenomenologically, it is described in terms of a photoelectric instability. It seems likely that these different observations are related, and therefore presumably the low-field effect will depend in part on the symmetry of the crystal! There are two obvious experiments. First, does the effect depend on the direction of the electric field relative to the crystallographic axes, e.g. is the effect reduced when the field lies in the basal plane? Large fields can still develop, but they will be in domains of different field directions. Secondly, what are the actual internal electric fields (as opposed to the small applied field)? Can one use the effects of the internal fields on the sharp $\mathrm{Cr} R$ lines to measure the real internal fields which are biasing defect reactions?

So far as applications are concerned, there is evidence that the degradation in resistivity saturates at a level which may well he satisfactory for fusion insulator applications (Dr Y. Chen, private communication).

\subsection{Ion-assisted coatings and radiation-enhanced adhe- sion}

Ion-assisted coating methods have the potential both to improve the quality of a coating and to prepare novel coatings with layered textures. The method has three main advantages $[85,86]$. Firstly, it is a low-temperature process, which can be used for deposition even on polymers which are not stable at higher temperalures. Secondly, good adhesion is achieved. There are several reasons for this: the process encourages intimate contact of coating and substrate; the cleaning of the substrate, and the generation of charged defects or of radicals can help (see below). Thirdly, properties can bc modificd by controlling the applied voltage and the relative arrival rates of metal (e.g. Ti to form TiN) and bombarding ion (e.g. Ar). Such properties include 
electrical, like sheet resistance, and mechanical. Indeed, novel mechanical properties can be demonstrated, an example being the multilayered TiN system in which successive layers were grown with different voltages applied [87]. In the case of TiN, the presence of oxygen can degrade propertics, apparently because of the formation of an oxynitride or dioxide.

The main effects of the ion beam are threefold. Firstly, momentum transfer helps to compact the surface. Secondly, the energy supplied to surface atoms helps to make them mobile, so again compaction of the surface is encouraged. The combination of energy-dependent and momentum-dependent factors favours low energy ions (e.g. $500 \mathrm{eV} \mathrm{Ar}$ ) rather than the energies of a few eV for bucket sources. The third factor is also momentum-dependent: damage is produced, and ions (like Ar) can be incorporated; again, low energies are favoured.

Radiation-enhanced adhesion is widely reported but rarely well controlled. However, the nature of the phenomenon is becoming far clearer, partly because the nature of adhesion is itself now better understood. The manner of enhancement is very varied: what happens for organics on metals is not the same as for metals on ionics or for metal on metal. In none of the cases I shall discuss is ion mixing across the boundary a factor.

For an organic (e.g. polyimide) on a clean metal surface, there is a degree of chemical interaction. 'This interaction can be enhanced by processes which generate free radicals, which themselves interact chemically with the metal atoms $[88,89]$. But this is only a part of the story of enhanced adhesion. In fact, the work of adhesion can be several orders of magnitude larger than that necessary to break all the bonds at the surface. The work of adhesion is dominated by the work to break the many weak links between polymer molecules as they are extraced from the film. The cross-linking caused by irradiation can be the largest effect in enhanced adhesion; one would expect enhancement when there is cross-linking, but negligible benefit when scission and degradation occurs. Certainly any gas release can worsen adhesion because of gas bubble formation at the interface. However, radiation of the organic prior to metal deposition may give rise to a texture which allows the metal and organic to interlock, so improving adhesion; in this case gas release may be helpful.

For a non-reactive metal (say $\mathrm{Au}$, or perhaps $\mathrm{Ag}$ or $\mathrm{Cu}$ ) on an oxide, the dominant term in the energy is electrostatic, the so-called image interaction [90-92] in which the ionic charges interact with their polarisation charges induced in the metal. What radiation does here is create charged defects, which lead to a larger adhesion (it is essentially the same effect which enables non-stoichiometric oxides like $\mathrm{NiO}$, chromia and ura- nia to be wetted more readily by liquid metals than very similar stoichiometric oxides like $\mathrm{MgO}$, alumina and thoria).

For two metals in contact, the story is quite different. Whilst simple experiments seem to suggest that $\mathrm{Au} / \mathrm{Ta}$ bonding can occur, this proves to be associated with poorly-cleaned surfaces. Once native oxide and adsorbate and contaminant layers have been removed, the effect vanishes. The effects seen when oxide is present are consistent with a process in the oxide akin to track formation; there is a threshold energy density, possibly associated with ionisation-induced interface phenomena [93]. Yet there are circumstances in which metal-metal bonds might be modified [94]. An example is $\mathrm{Cu}$ / carbide, where the transition-metal carbide is itself a metal. The interfacial energy depends on the carbon stoichiometry, and a radiation-induced alteration in carbon fraction would change adhesion.

\subsection{Nanowriting}

High precision drilling by excimer lasers is already established. But high precision here means an ablation process, achieving $50 \mu \mathrm{m}$ holes in a ceramic like alumina. Nanowriting [95-98] using electron beams (with perhaps $100 \mathrm{keV}$ energy, less than that needed for knock-on damage) drills holes four orders of magnitude smaller. Thus Turner et al. drill a hole in a $65 \mathrm{~nm}$ $\mathrm{MgO}$ cube; with a $0.5 \mathrm{nA}$ current and $2.5 \mathrm{~nm}$ diameter beam, the hole develops at about $0.5 \mathrm{~nm} / \mathrm{s}$ (the speed is roughly linear in the current, but there is a threshold in some cases). What is also surprising is that the hole develops from both the beam entrance side and the beam exit side of the microcrystal. This behaviour varies from crystal to crystal: for $\mathrm{MgO}$ and $\mathrm{Si}$, the hole develops most strongly at the beam exit; for alumina an oxygen bubble develops internally and then bursts; for beta alumina, indentations grow from the front surface; for $\mathrm{Al}$, holes develop mainly from the front. There are also some unusual effects at the beam edges.

What might be happening? Clearly there is some desorption process, though whether it is KnotekFeibelman or Menzel-Gomer-Redhead is not clear (and not necessarily the same in all crystals). Evaporation from favoured sites and momentum-assisted diffusion may have a role. The electric fields developed during irradiation will certainly be important. The process is relatively efficient in electron numbers (if not in energy) rcmoving onc $\mathrm{MgO}$ unit for every few 1000 electrons.

One of the most promising methods for resolution below $1 \mathrm{~nm}$ in electron beam lithography is indeed the direct removal of sublimed films of aluminium fluoride or alumina by a beam which can be as small as $0.5 \mathrm{~nm}$ wide. Whilst $1 \mathrm{~nm}$ structures have been fabricated, they have still to be turned into useful devices. For 
organics, like the traditional PMMA (the highest-resolution electron resist), the resolution appears to be fixed by the way the damage occurs locally in the molecule, rather than the overall size (molecular weight) of the molecule itself. For positive tone lithography, the beam causcs scission, and the shorter segments are dissolved; for negative tone lithography, the beam causes cross-linking, and again the shorter segments are removed.

\section{Closing comments}

I have not yet touched on new methods of investigating radiation damage, such as scanning tunnelling microscopy, though early studies suggest considerable potential [99-101]. Perhaps more importantly, I have said relatively little about modelling. Yet this will surely be a major force in the future. The developments in hardware and software mean cascades can be handled in molecular dynamics (at least in simple cases); the methods to handle quantum chemistry at the same time removes further restrictions. Yet the role of excited states, the localisation of energy, the role of precursors, and the complexity of some of the systems of importance (notably as regards the evolution of microstructure and the inhomogeneity which needs Poisson or percolation methods to analyse) will leave plenty of challenges.

\section{Acknowledgements}

I am grateful to Dr. R.G.J. Ball, Dr. S.J. Bull, Dr. Y. Chen and Dr. G.P. Pells for discussions of their work prior to publication.

\section{References}

[1] See e.g.: E. Balanzat and S. Bouffard, Solid State Phenom. 30/31 (1993) 7 (especially p. 64).

[2] See e.g.: J. Delaplace, Solid State Phenom. 30/31 (1993) 169.

[3] W. Hayes and A.M. Stoneham, Defects and Defect Processes in Non-metallic Solids (Wiley, New York, 1985).

[4] L.W. Hobbs, A.E. Hughes and D. Pooley, Proc. R. Soc. A 332 (1973) 167.

[5] P.M. Petroff and L.C. Kimerling, Appl. Phys. Lett. 29 (1976) 461.

[6] R.W. Grimes, C.R.A. Catlow and A.M. Stoneham, J. Amer. Ceram. Soc. 72 (1989) 1856.

[7] A.M. Stoneham and M.J.L. Sangster, Philos. Mag. B 43 (1980) 609.

[8] A.M. Stoneham and M.M.D. Ramos, J. Solid State Chem. 106 (1993) 2.

[9] T.C. Choy and A.M. Stoneham, Europhys. Lett. 23 (1993) 99.
[10] N. Itoh, A.M. Stoneham and A.H. Harker, J. Phys. C 10 (1977) 4197.

[11] K.S. Song and R.T. Williams, Self-Trapped Excitons, Springer Series in Solid State Science, vol. 105 (Springer, Berlin, 1993).

[12] W.B. Fowler and N. Itoh, Atomic Processes Induced by Electronic Excitation in Non-Metallic Solids (World Scientific, Singapore, 1990).

[13] G.P. Pells, AEA-RS-2271 (1992).

[14] G.P. Pells, Schloss Ringberg Conf. (1993).

[15] R.H. Magruder, Li Yang, R.F. Haglund, C.W. White, Lina Yang, R. Dorsinville and R.R. Alfano, Appl. Phys. Lett. 62 (1993) 1730.

[16] E.g.: G. Dearnaley, Mater. Technol. 8 (1993) 4.

[17] A.M. Stoneham and L.W. Smith, J. Phys. Cond. Mater. 3 (1991) 225.

[18] J.M. Stevels, Progress in the Theory of the Physical Properties of Glass (Elsevier, 1944);

Medium range order discussed by S.R. Elliott, Nature 354 (1991) 445 , may well be partly due to the oxygenoxygen interactions implied by Stevels.

[19] G. Tinivella and A.M. Stoneham, UKAEA Report M3142 (1980).

[20] M. Antonini, A. Manara and P. Lensi, Properties of Silicon Dioxide, ed. S.T. Pantiledes (New York,1978) p. 316.

[21] A. Navrotsky, Diff. Diff. Data 53/54 (1978) 61.

[22] P.G. Klemens, F.W. Clinard and R.J. Livak, J. Appl. Phys. 62 (1987) 3953.

[23] J. Lindhard, V. Nielsen, M. Scharff and P.V. Thomsen, K. Dan. Vidensk Selsk Mat Fys. Medd. 33 (10) (1963).

[24] A.M. Stoneham, Nucl. Instr. and Meth. B 48 (1990) 389.

[25] M. Cini, Solid State Commun. 20 (1975) 605.

[26] A.L. Shluger and A.M. Stoneham, J. Phys. Condens. Mat. 5 (1993) 3049.

[27] K. Ong, K.S. Song, R. Monnier and A.M. Stoneham, J. Phys. C 12 (1979) 4649.

[28] M. Bode, A. Ourmazd, J. Cunningham and M. Hong, Phys. Rev. Lett 67 (1991) 843.

[29] R. Kadano, A. Matsushita and K. Nagamine, Phys. Rev. Lett. 67 (1991) 3689.

[30] R. Kadano, A. Matsushita, K. Nishiyama and K. Nagamine Phys. Rev. B 46 (1992) 8586.

[31] S.F.J. Cox and C. Schwab (1993) preprint for Maue Muon meeting.

[32] A.M. Stoneham, Cryst. Latt. Def. Amorph. Mat. 14 (1987) 173.

[33] M.A. Schildbach and A.V. Hamza, Phys. Rev. B 45 (1992) 6197.

[34] M.L. Knotek, Rep. Prog. Phys. 47 (1984) 1499.

[35] U. Stawinski and E. Bauer, Phys. Rev. B 47 (1993) 12820.

[36] J. Kanasaki, A. Okano, K. Ishikawa, Y. Nakai and N. Itoh, Phys. Rev. Lett. 70 (1993) 2495.

[37] K. Hattori, A. Okano, Y. Nakai and N. Itoh, Phys. Rev. B 45 (1992) 8424; K. Hattori, A. Okano, Y. Nakai, N. Itoh and R.F. Haglund, J. Phys. Condens. Mat. 3 (1991) 7001; C.K. Ong, G.S. Khoo, N. Itoh and K. Hattori, Surf. Sci. 259 (1991) L787;

N. Itoh, K. Hattori, Y. Nakai, J. Kanasaki, A. Okaro, 
C.K. Ong and G.S. Khoo, Appl. Phys. Lett. 60 (1992) 3271.

[38] S. Sugden, C.J. Sofield and M.P. Murrell, Nucl. Instr. Meth. B 67 (1992) 5.

[39] A.-M. Lanzillotto, T.E. Madey and R.A. Baragiola, Phys. Rev. Lett. 67 (1991) 232.

[40] C. Fiori and R.A.B. Devine, Phys. Rev. Lett. 52 (1984) 2081.

[41] R.E. Johnson and B.U.R. Sundqvist, Physics Today (1992) 28.

[42] A. Okano, A.Y. Matsuura, K. Hattori and N. Itoh, J. Appl. Phys. 73 (1993) 3158.

[43] A.J. Fisher, A.M. Stoneham and W. Hayes, Phys. Rev. Lett. 64 (1990) 2667;

A.J. Fisher, A.M. Stoneham and W. Hayes, J. Phys. Condens. Mat. 2 (1990) 6707.

[44] P.N. Saeta and B.I. Greene, Phys. Rev. Lett. 70 (1993) 3588.

[45] F. Bridges, G. Davies, J. Robertson and A.M. Stoneham, in: 1990 Current Issues in Condensed Matter Spectroscopy (Institute of Physics, Bristol).

[46] C. Itoh, T. Suzuki and N. Itoh, Phys. Rev, B 41 (1990) 3794.

[47] F.L. Galeener, D.B. Kerwin, A.J. Miller and J.C. Mikkelsen Phys. Rev. B 47 (1993) 7760.

[48] D.H. Lcvy, K.K. Glcason, M. Rothschild and J.H.C. Sedlacek, J. Appl. Phys. 73 (1993) 2809.

[49] R.C. Barklie, T.J. Ennis, K. Reeson and P.L.F. Hemment, J. Phys. Condens. Mat. 3 (1991) 2115.

[50] F.B. McLean, JEEE Trans. Nucl. Sci. NS-27 (1980) 1651.

[51] P.S. Winokur, H.E. Boesch, J.M. McGarrity and F.B. McLean, J. Appl. Phys. 50 (1979) 3492.

[52] See e.g.: J.-L. Leray, Solid State Phenom. 30/31 (1993) 387.

[53] C.H. de Novion and A. Barbu, Solid State Phenom. 30/31 (1993) 277 (especially p. 288).

[54] D.L. Griscom, J. Non. Cryst. Solid 73 (1985) 51; D.L. Griscom, SPIE 541 (1985) 38.

[55] R.G.J. Ball and R.W. Grimes, Phil. Mag. A 66 (1992) 473.

[56] R.G.J. Ball and R.W. Grimes, J. Nucl. Mat. 188 (1992) 216.

[57] H.-J. Matzke, J. Mat. Sci. 2 (1967) 444.

[58] R. Linder and H.-J. Matzke, Z. Natur a 14 (1959) 1074.

[59] G.P. Summers, E.A. Burke, D.B. Chrisey, M. Nastasi and J. Tesmer, Appl. Phys. Lett. 55 (1989) 1469.

[60] Y. Momose, Y. Tamura, M. Ogino, S. Okazaki and M. Hirayama, J. Vac. Sci. Tech. A 10 (1992) 229.

[61] K.T. Gillen and R.L. Clough, Polymer 33 (1992) A358.

[62] J. Davinas, Solid State Phenom. 30/31 (1993) 317.

[63] T. Venkatesan, L. Calcagno, B.S. Elman and G. Foti, in: Ion Beam Modification of Materials 2, eds. P. Mazzoldi and G.W. Arnold (Elsevier, Amsterdam, 1987).

[64] B. Wasserman, Phys. Rev. B 34 (1986) 1926.

[65] M. Toulemonde and F. Studer, Solid State Phenom. 30/31 (1993) 477.

[66] E. Dartyge and P. Sigmund, Phys. Rev. B 32 (1985) 5429.

[67] N. Chou, A.D. Marwick, R.D. Goldblatt, Leping Li, G. Coleman, J.E. Heidenreich and J.R. Paraszczak, J. Vac. Sci. Tech. A 10 (1992) 248.
[68] K. Nakamac, K. Yamaguchi, S. Tanigawa, K. Sumiya and I. Matsumoto, J. Chem. Soc. Japan 11 (1987) 1995.

[69] D.J. Krajnovich and J.E. Vaquez, J. Appl. Phys. 73 (1993) 3001.

[70] E.R. Hodgson, Cryst. Latt. Defects Amorph. Mat. 18 (1989) 169

[71] E.R. Hodgson, J, Nucl. Mat. 179-181 (1991) 383.

[72] E.R. Hodgson, Rad. Eff. 119-121 (1991) 827.

[73] E.R. Hodgson, Nucl. Instr. and Meth. B 65 (1992) 298.

[74] E.R. Hodgson, J. Nucl. Mat. 191-194 (1992) 552.

[75] G.P. Pells, J. Nucl. Mat. 184 (1991) 177.

[76] V.M. Ivanov, G.M. Kalinin, V.F. Kuzovitikin, S.P. Sklizkov, N.V. Morkina, V.V. Sarksyan and V.A. Skobeleva, Inorg. Mat. 17 (1981) 1203.

[77] N. Itoh, T. Tanaka and F.W. Clinard, J. Nucl. Mat. 183 (1991) 25.

[78] A.S. Portnyagin, I.I. Milman and V.S. Kortov, Sov. Phys. Solid State 33 (1991) 1272.

[79] A.S. Portnyagin, I.I. Milman and V.S. Kortov (1992) Sov. Phys. Solid State (in press).

[80] G.P. Pells, to be published.

[81] R. Bullough and A.D. Brailsford, J. Nucl. Mat. 44 (1972) 121

[82] U. Jain and A.B. Lidiard, Phil. Mag. 35 (1975) 245.

[83] A. Perez and C.I. McHargue, Solid State Phenom. 30/31 (1993) 297.

[84] S.A. Basun, A.A. Kaplyanski and S.P. Feofilov, Sov. Phys. JETP 60 (1984) 1182.

[85] S.J. Bull, in: Advanced Technology for Surface Engineering (CEC Eurocourse on Interfaces and Adhesion) eds. W. Gissler and H. Jehn (Kluwer, Dordrecht, 1992) pp. 31-68.

[86] G. Dearnaley and R.K. Saini, Surf. Coat. Technol. 43/44 (1990) 779 .

[87] S.J. Bull and A.M. Jones, Surf. Coat. Technol. (1993) in press.

[88] A.M. Stoneham, M.M.D. Ramos and A.P. Sutton, Phil. Mag. A 67 (1993) 797.

[89] M.M.D. Ramos, A.P. Sutton and A.M. Stoneham, Acta. Met. Mater, 41 (1993) 2105.

[90] A.M. Stoneham and P.W. Tasker, J. Phys. C 18 (1985) L543.

[91] A.M. Stoneham and P.W. Tasker, in: Ceramic Microstructures 86 ed. J.A. Pask (Plenum, New York, 1987) p. 155.

[92] D.M. Duffy, J.H. Harding and A.M. Stoneham, Phil. Mag. A 67 (1993) 865.

[93] (a) S. Sugden, C.J. Sofield and M.P. Murrell, Nucl. Instr. and Meth. B 67 (1992) 452;

(b) S. Sugden, C.J. Sofield and M.P. Murrell, Nucl. Instr. and Meth. B 44 (1989) 137;

(c) S. Sugden, C.J. Sofield and M.P. Murrell, Rad. Eff. 115 (1990) 79.

[94] A.M. Stoneham, D.M. Duffy, J.H. Harding and P.W. Tasker, AEA Report InTec 0708 (1991), Designing Interfaces II, ed. S.D. Peteves (1993) p. 573.

[95] C.J. Humphreys, I.G. Salisbury, S.D. Berger, R.S. Timsit and M.E. Mochel, Inst. Phys. Conf. Ser. 78 (1985) 1.

[96] P.S. Turner, T.J. Bullough, R.W. Devenish, D.M. Maher and C.J. Humphreys, Phil. Mag. Lett. 61 (1990) 181.

[97] C.J. Humphreys, T.J. Bullough, R.W. Devenish, D.M. Maher and P.S. Turner, Scanning Microsc. Suppl. 4 (1990) 185. 
[98] T.J. Bullough, C.J. Humphreys and R.W. Devenish, MRS Proc. 157 (1990) 323.

[99] T. Schimmel, H. Fuchs, R. Sander and M. Lux-Steiner, Ultramicroscopy 42/44 (1992) 683;

see also: H. Fuchs and T. Schimmel, Adv. Mater. 3 (1991) 112.
[100] D. Snowden-Ifft, P.B. Price, L.A. Nagahara and A. Fujishima, Phys. Rev. Lett. 70 (1993) 2348.

[101] M.J. Novakowiski, J.M. Vohs and D.A. Bonnell, J. Amer. Ceram. Soc. 76 (1993) 279. 